\title{
Targeting myeloid-derived suppressor cells augments antitumor activity against lung cancer
}

REVIEW

This article was published in the following Dove Press journal:

ImmunoTargets and Therapy

II October 2012

Number of times this article has been viewed

Minu K Srivastava ${ }^{1,2}$

Li Zhu',2

Marni Harris-White ${ }^{2}$

Min Huang ${ }^{1-3}$

Maie St John ${ }^{1,3}$

Jay M Lee ${ }^{1,3}$

Ravi Salgia ${ }^{4}$

Robert B Cameron ${ }^{1,3,5}$

Robert Strieter 6

Steven Dubinett ${ }^{1-3}$

Sherven Sharma ${ }^{1-3}$

'Department of Medicine, UCLA Lung

Cancer Research Program, David

Geffen School of Medicine at UCLA,

Los Angeles, CA, ${ }^{2}$ Molecular Gene

Medicine Laboratory, Veterans Affairs

Greater Los Angeles Healthcare

System, Los Angeles, CA, ${ }^{3}$ Jonsson

Comprehensive Cancer Center,

David Geffen School of Medicine at

UCLA, Los Angeles, CA, ${ }^{4}$ Department of Medicine, University of Chicago,

Chicago, IL, ${ }^{5}$ Department of Surgery,

Veterans Affairs Greater Los Angeles Healthcare System, Los Angeles, CA, ${ }^{6}$ Department of Medicine, University of Virginia, Charlottesville, VA, USA

Correspondence: Sherven Sharma Molecular Gene Medicine Laboratory, Veterans Affairs Greater Los Angeles Healthcare System, Los Angeles, CA 90073, USA

$\mathrm{Tel}+\mathrm{I} 3$ I 047837 II ext 4I863

$\mathrm{Fax}+\mathrm{I} 3102684807$

Email ssharma@mednet.ucla.edu
Abstract: Lung cancer evades host immune surveillance by dysregulating inflammation. Tumors and their surrounding stromata produce growth factors, cytokines, and chemokines that recruit, expand, and/or activate myeloid-derived suppressor cells (MDSCs). MDSCs regulate immune responses and are frequently found in malignancy. In this review the authors discuss tumor-MDSC interactions that suppress host antitumor activities and the authors' recent findings regarding MDSC depletion that led to improved therapeutic vaccination responses against lung cancer. Despite the identification of a repertoire of tumor antigens, hurdles persist for immunebased anticancer therapies. It is likely that combined therapies that address the multiple immune deficits in cancer patients will be required for effective therapy. MDSCs play a major role in the suppression of T-cell activation and they sustain tumor growth, proliferation, and metastases. Regulation of MDSC recruitment, differentiation or expansion, and inhibition of the MDSC suppressive function with pharmacologic agents will be useful in the control of cancer growth and progression. Pharmacologic agents that regulate MDSCs may be more effective when combined with immunotherapies. Optimization of combined approaches that simultaneously downregulate MDSC suppressor pathways, restore APC immune-stimulating activity, and expand tumor-reactive $\mathrm{T}$ cells will be useful in improving therapy.

Keywords: MDSCs, antigen-presenting cells, natural killer cell activation, T-cell activation, immunotherapy

\section{Introduction}

Lung cancer is a challenging health problem, with more than 1.1 million deaths attributed to lung cancer worldwide each year. ${ }^{1}$ With current therapy, the long-term survival rate for the majority of lung cancer patients is low; thus, new therapeutic strategies are needed. Immunotherapy is an option; however, activation of the immune system alone is not sufficient for antitumor activity. Combined therapies that boost immune activation and reverse mechanisms of immune suppression ultimately will prove beneficial in the fight against lung cancer.

Modifications in oncogenes and tumor suppressor genes and/or epigenetic changes lead to tumor progression and local tissue invasion that cause the persistence of inflammatory cellular tumor infiltrates. The tumor causes the cellular infiltrates to sustain dysregulated inflammation, which immunologically is unresponsive to the tumor. Characterization of the infiltrate-tumor interactions may aid in the design of specific personalized anticancer treatments by reprogramming the tumor microenvironment. A significant proportion of the inflammatory cellular tumor infiltrates are myeloid-derived suppressor cells (MDSCs), which maintain an 
immunosuppressive environment, ${ }^{2-4}$ supporting tumor expansion in the surrounding tissue and at distant sites by facilitating angiogenesis and metastases.

\section{MDSCs and the tumor microenvironment}

MDSCs are a heterogeneous population of bone marrowderived cells comprising myeloid precursors of macrophages, granulocytes, and dendritic cells (DCs). In mice, MDSCs are identified by the cell surface expression of Gr1 and CD11b. Recently, MDSCs have been subcategorized based on the expression of Ly6C and Ly6G within the $\mathrm{Gr} 1$ cell population. CD $11 \mathrm{~b}^{+}$Ly $6 \mathrm{G}^{+}$Ly $6 \mathrm{C}^{\mathrm{lo}}$ cells with multilobed nuclei and granulocytic-like morphology are called granulocytic MDSCs, and CD $11 b^{+} \mathrm{Ly}_{6 \mathrm{G}^{-}} \mathrm{Ly} 6 \mathrm{C}^{\mathrm{hi}}$ cells are referred to as monocytic MDSCs. ${ }^{5}$ In humans, MDSCs are identified by surface expression of CD33, and the lack of expression of markers of mature myeloid and lymphoid cells. They are typically $\mathrm{CD} 11 \mathrm{~b}^{+} \mathrm{CD} 33^{+} \mathrm{CD} 34^{+} \mathrm{CD} 14^{-}$and vary in CD15, CD124, CD66, and major histocompatibility complex (MHC) class II expression. ${ }^{6}$ Melanoma MDSCs express $\mathrm{CD}_{14}{ }^{+} \mathrm{CD} 11 \mathrm{~b}^{+} \mathrm{HLA}-\mathrm{DR} \mathrm{R}^{\mathrm{lo} / \mathrm{neg}},{ }^{7}$ and $\mathrm{CD} 11 \mathrm{~b}^{+} \mathrm{CD} 14^{-} \mathrm{CD} 15^{+} \mathrm{CD} 33^{+} \mathrm{MDSC}$ have been described in non-small cell lung cancer patients. ${ }^{8}$ Because MDSC gene expression varies in different tumor types, the identification of a unique set of markers for human MDSCs has been challenging. Thus, phenotypic characteristics and the functional ability to suppress $\mathrm{T}$ cells are used to define MDSCs.

MDSC accrual, expansion, and activation in the tumor are dependent on tumor- or stromal-derived growth factors, cytokines, and chemokines. These include prostaglandin E2 (PGE2), matrix metalloproteinases, transforming growth factor beta (TGF- $\beta$ ), interleukin (IL)-10, vascular endothelial growth factor (VEGF), IL-1 beta (IL-1 $\beta$ ), IL-4, granulocyte-macrophage colony-stimulating factor (GM-CSF), IL-6, IL-13, S100A8/A9, stem cell factor, CCL2, CXCL5, CXCL12, toll-like receptor agonists, and tumor-derived HSP72. ${ }^{9-11}$ GM-CSF supports the survival and expansion of MDSCs in the tumor microenvironment. ${ }^{12}$ The sources of GM-CSF include tumors and activated immune effectors such as T cells, natural killer (NK) cells, and DCs. IL- $1 \beta$ recruits MDSCs; mice inoculated with tumor cells secreting IL-1 $\beta$ exhibit elevated levels of MDSCs. IL-1 $\beta$ induced MDSCs have elevated levels of reactive oxygen species with enhanced T-cell suppression. ${ }^{13}$ Furthermore, IL-6 secreted by tumors increases the frequency of MDSCs in tumors. ${ }^{14}$ Proinflammatory S100 proteins also cause MDSC accumulation and immune suppression. ${ }^{15,16}$ The S100A8/A9 proteins are members of a large family of inflammatory and noninflammatory proteins produced by neutrophils, activated monocytes, tumor cells, and MDSCs. MDSCs have receptors for S100A8/A9 complexes and exhibit autocrine enhancement of S100A8/A9 levels. Antibody receptor blockade reduces the MDSC frequency in tumorbearing mice. Mice that are genetically S100A9 deficient are resistant to tumor challenge but become susceptible if MDSCs are adoptively transferred from wild-type mice. S100A8/A9 proteins affect MDSCs through two known mechanisms: (1) they block the further differentiation of myeloid precursors to differentiated DCs and macrophages through a signal transducer and activator of transcription 3-dependent mechanism and (2) they increase tumor MDSCs through a nuclear factor kappa-light-chain-enhancer of activated B cells-dependent pathway. ${ }^{15,16}$ This suggests that S100 protein-mediated accumulation of tumor MDSCs may serve as a useful therapeutic target to reduce immune suppression. Inflammation-mediated S100A8/A9 induction, MDSC accumulation, and the resulting immune suppression may represent a common mechanism of cancer-related escape from immune surveillance.

\section{MDSC mechanisms of immune modulation}

MDSCs suppress T-cell activation through several mechanisms (Figure 1). MDSCs exhibit control of T-cell responses via two enzymes: (1) arginase-1 (ARG1), which depletes L-arginine, and (2) inducible nitric oxide synthase 2 (iNOS), which generates nitric oxide. ${ }^{17}$ TGF- $\beta$ and IL-10 induce MDSC ARG1, which suppresses CD4 and CD8 T-cell activation. Interferon gamma (IFN- $\gamma$ ) and tumor necrosis factor alpha induce MDSC iNOS, which releases nitric oxide and induces T-cell apoptosis. ${ }^{18}$

MDSCs also deplete cysteine, an essential amino acid for T-cell activation. ${ }^{19} \mathrm{~T}$ cells obtain their cysteine from extracellular sources (specifically, DCs and macrophages), which convert methionine to cysteine and reduce cystine to cysteine during normal antigen processing and presentation. MDSCs depend on the conversion of cystine to cysteine. In the tumor microenvironment large numbers of MDSCs deplete the available cysteine, which deprives DCs and macrophages of this important molecule and indirectly lowers DC and macrophage production of cysteine and T-cell activation. ${ }^{19}$

MDSC-mediated downregulation of T-cell L-selectin (CD62L) impairs T-cell migration and activity. ${ }^{20} \mathrm{CD} 62 \mathrm{~L}$ is a plasma membrane molecule necessary for the homing of naive 


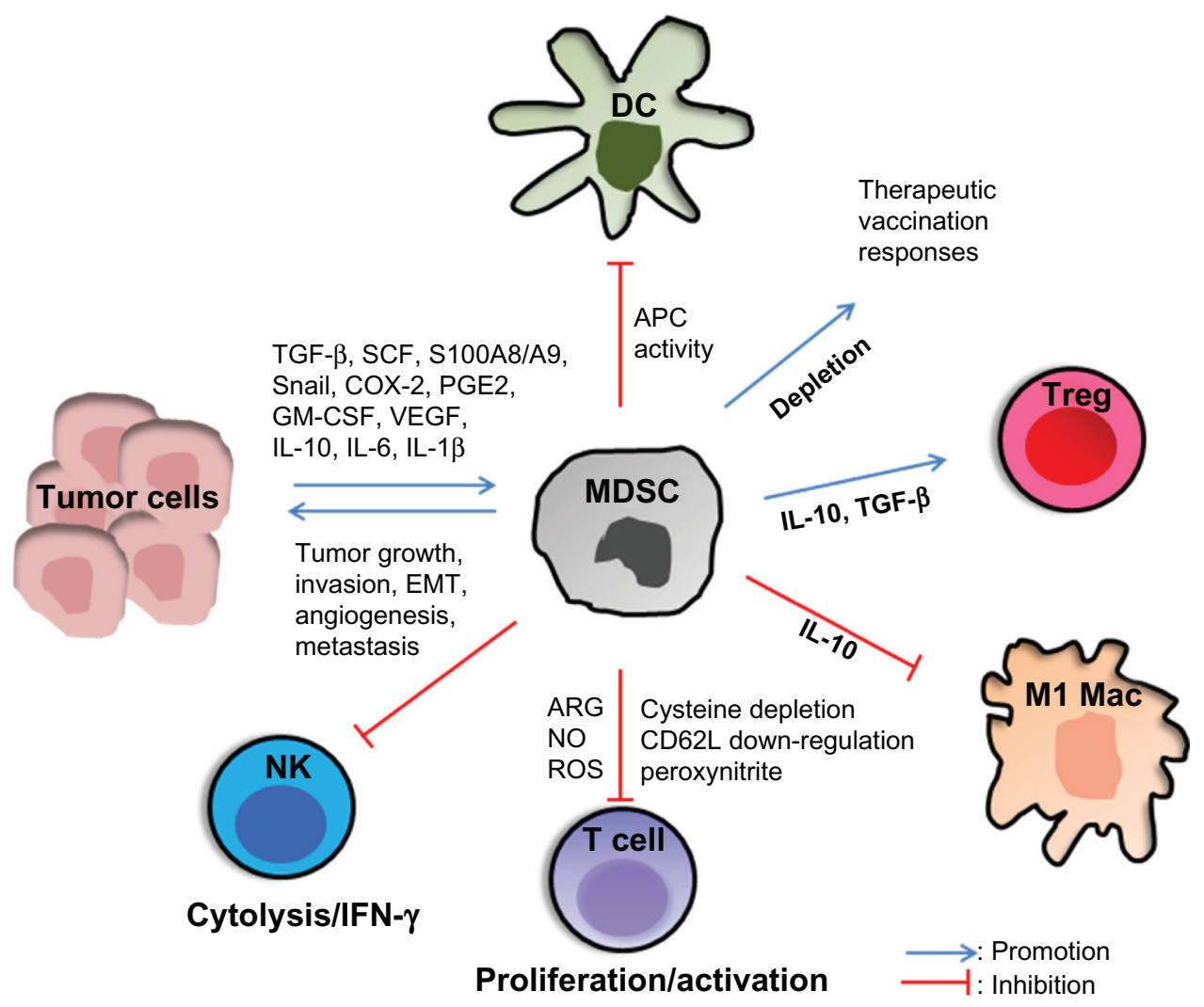

Figure I Myeloid-derived suppressor cells (MDSCs) suppress antitumor activity.

Notes: MDSCs are recruited and expanded in the tumor through the induction/production of transforming growth factor beta (TGF- $\beta$ ), stem cell factor (SCF), cyclooxygenase 2 (COX-2), prostaglandin E2 (PGE2), interleukin (IL)-6, granulocyte-macrophage colony-stimulating factor (GM-CSF), IL-I0, IL-I beta (ILI- $\beta$ ), vascular endothelial growth factor (VEGF), SI00 proteins, and Snail. T-cell activation is suppressed by MDSC-mediated deprivation of L-arginine and cysteine from the environment, production of reactive oxygen species (ROS) and peroxynitrite, downregulation of L-selectin (CD62L) and the T-cell receptor-associated zeta chain, and the induction of T regulatory (Treg) cells through MDSC IL- 10 and TGF- $\beta$ production. MDSCs suppress natural killer (NK) cell cytotoxicity and NK interferon gamma (IFN- $\gamma$ ) production and they inhibit MI macrophages (MI Macs). MDSC expansion and IL-I0 production inhibit dendritic cell (DC) antigen presentation. MDSC depletion enhances therapeutic vaccination responses.

Abbreviations: APC, antigen-presenting cell; ARG, arginase; EMT, epithelial-mesenchymal transition; NO, nitric oxide.

T cells to lymph nodes for activation; MDSC downregulation of $\mathrm{CD} 62 \mathrm{~L}$ on naive $\mathrm{T}$ cells reduces this process. ${ }^{20}$

The MDSC release of reactive oxygen species and peroxynitrite inhibits $\mathrm{CD} 8^{+} \mathrm{T}$ cells by catalyzing the nitration of the T-cell receptor, thereby preventing T-cellpeptide-MHC interactions. ${ }^{21}$ MDSCs also downregulate the T-cell receptor-associated zeta chain ${ }^{22}$ required for T-cell activation. MDSCs directly induce $\mathrm{T}$ regulatory (Treg) cells through the production of IL-10, TGF- $\beta$, or ARG $1 .{ }^{10}$ The Treg cells actively downregulate the activation and expansion of antitumor reactive $\mathrm{T}^{23}$ and NK cells. ${ }^{24}$ MDSCs polarize $\mathrm{T}$ cells toward a type 2 tumor-promoting phenotype by IL-10 production and downregulation of macrophage IL-12. ${ }^{25}$

T-cell nonresponsiveness to tumor antigens has been shown to be an early event in tumor progression. ${ }^{26}$ Although T-cell tolerance in cancer has been shown to be mediated by host antigen-presenting cells (APCs) ${ }^{27}$ the nature of these APCs has not been clear. Recent evidence suggests that
MDSCs may represent a population of APCs responsible for induction of antigen-specific CD8 T-cell tolerance in cancer. ${ }^{21} \mathrm{CD} 8 \mathrm{~T}$-cell responses in vivo are induced by professional APCs that present exogenous antigens in a MHC class I-restricted manner. ${ }^{28}$ This has been referred to as cross-priming or representation. DCs are the host APCs responsible for cross-priming by presenting epitopes obtained from apoptotic cells. However, in tumor-bearing hosts, there is a state of T-cell unresponsiveness that is regulated by DCs. The tumor not only fails to provide the inflammatory signals needed for efficient DC activation but also inhibits DC differentiation and maturation through IL- $10^{29}$ and VEGF. ${ }^{30}$ Myeloid precursor differentiation into granulocytes, macrophages, and DCs is frequently dysregulated in the tumor, leading to an accumulation of MDSCs. Immature DCs have little or no expression of costimulatory molecules such as CD80, CD86, and CD40 on their surface and they produce little or no IL-12, which is required to support T-cell proliferation and for leading to tolerance. MDSCs also impair 
innate NK cells by inhibiting their cytotoxic ability and IFN- $\gamma$ production. $^{31}$

\section{Lung cancer genetic signatures that drive immune suppression}

The authors' laboratory has been evaluating tumor signatures that maintain tumor growth through the modulation of immune activity. The authors have reported that the constitutive overexpression of tumor cyclooxygenase 2 (COX-2) leads to an immunosuppressive network in lung cancer. ${ }^{32,33}$ Overexpression of tumor COX-2 is associated with decreased host immunity ${ }^{32-34}$ and metastasis. ${ }^{35} \mathrm{COX}-2$ has been shown to modulate MDSC activity in lung cancer through the production of high levels of ARG1, which blocks T-cell function. ${ }^{36}$ Until recently, the mechanism that induces ARG1 in MDSCs was unknown. Utilizing mice bearing the Lewis lung carcinoma (3LL), Rodriguez et $\mathrm{al}^{36}$ showed that maintenance of MDSC ARG1 expression was independent of T-cell cytokine but was dependent on tumor-derived PGE2. These results demonstrated a new pathway of prostaglandin-induced immune dysfunction and provided a novel mechanism to explain the antitumor benefits of COX-2 inhibitors. The present authors have demonstrated that the tumor microenvironment induced DC immunosuppressive effects that promoted tumor growth and that tumor COX-2 inhibition restored DC APC and antitumor activity. ${ }^{34}$

The complex interactions between MDSCs and Treg cells are yet to be fully defined; however, it is evident that MDSCs promote Treg cell development in vivo. Tumor-reactive $\mathrm{T}$ cells are found in lung cancer tissue but fail to respond to tumor because of $\mathrm{CD} 4{ }^{+} \mathrm{CD} 25^{+}$Treg cells. ${ }^{37} \mathrm{CD} 4{ }^{+} \mathrm{CD} 25^{+}$ Treg cells play an important role in the maintenance of immunologic self-tolerance and in suppressing antitumor immune responses mediated via self-reactive lymphocytes. ${ }^{23}$ Reducing the number of Treg cells or abrogating their activity within the tumor environment could induce effective tumor immunity in otherwise nonresponding hosts by activating tumor-specific as well as nonspecific effector cells. Studies have demonstrated that tumor COX-2 expression contributes to decreased host antitumor immune responses by affecting the frequency and activity of $\mathrm{CD} 4^{+} \mathrm{CD} 25^{+} \mathrm{FOXP} 3^{+}$Treg cells. ${ }^{38,39}$ COX-2 inhibition reduced Treg cell frequency and activity, attenuated Foxp3 expression in tumor-infiltrating lymphocytes, and decreased tumor burden. Transfer of Treg cells or administration of PGE2 to mice receiving COX-2 inhibitors reversed these effects. ${ }^{38}$ These studies were the first documentation that COX-2 inhibition downregulated tumor-induced Treg cell activity, leading to the restoration of antitumor responses.
In addition to $\mathrm{COX}-2$, the present authors are currently evaluating the effect of genetic programs that regulate epithelial-mesenchymal transition (EMT) on tumor growth and metastases in lung cancer. Cancer cells acquire the ability to progress, invade, and metastasize by undergoing the process of EMT, by activating transcription factors (eg, Snail, Twist, Zeb, Slug) that repress epithelial cadherin. ${ }^{40,41}$ Snail expression in primary non-small cell lung cancer has been associated with a shorter overall survival, ${ }^{42}$ and in melanoma, Snail expression has been demonstrated to be important in EMT-induced metastases. ${ }^{43}$ The authors have demonstrated that tumor Snail expression alters tumor growth and metastasis by affecting MDSCs in the tumor microenvironment. ${ }^{44}$ Compared with controls, the tumors of mice implanted with 3LL Snail knockdown cells decreased MDSC frequency, activity, and intracellular expression of ARG1 but increased CD107a cytolytic marker expression on CD8 T cells. Snail knockdown tumor cells demonstrated reduced subcutaneous growth and lung metastases.

\section{MDSC depletion and antitumor activity in lung cancer}

Although immunotherapy for lung cancer has potential, to date there has been no randomized trial reporting documented survival benefits. ${ }^{45}$ Tumor-induced immune suppression through MDSCs could contribute to the limited efficacy of the approaches. In a recent study, the authors tested the hypothesis that activating immune cells through therapeutic vaccination and disruption of MDSC-mediated immune suppression would improve antitumor activity in a murine lung cancer model. ${ }^{46}$ To initiate antigen-specific responses, a cellular vaccine, comprising bone marrow adherent cells pulsed with the model ovalbumin (OVA) antigen expressed by OVA-modified 3LL cells, was utilized. The bone marrow adherent cells were pulsed with the OVA antigen to allow for antigen processing and presentation and were then injected subcutaneously into mice on the contralateral flank of the established tumor to initiate antigen-specific antitumor immune responses. To circumvent MDSC-mediated immune suppression, MDSCs were depleted with monoclonal antibodies (anti-Gr1 or anti-Ly6G).

Broad depletion by antibodies that targeted Gr1 or Ly6G on the MDSC population led to a decrease in MDSCs in the tumor and systemically in the blood, spleen, and bone marrow. The APC activity in the tumor was reduced as the tumor progressed; however, MDSC depletion led to increased frequency and activities of APC, NK cell, and T-cell effectors. Along with the increased NK and T-cell 
activities, there was an increase in the frequency of apoptotic tumor cells and a concomitant reduction in tumor burden and migration of tumor cells from the primary tumor site to the lung. The reduction in tumor growth and abrogation in tumor cell migration may be explained by an increase in the frequency of activated $\mathrm{T}$ and/or NK effector-mediated tumor apoptosis and/or T or NK IFN- $\gamma$-mediated antiangiogenesis. Following MDSC depletion, the antiangiogenic chemokines CXCL9 and CXCL10 were increased and the pro-angiogenic cytokines VEGF-A, angiopoietin 1, angiopoietin 2, CXCL2, and CXCL5 were markedly reduced. Accompanying this profile was a reduction in the endothelial markers MECA-32 in the tumor but there was also an increase in CXCR3 expression. MDSC depletion led to an influx of IFN- $\gamma$-producing activated $\mathrm{T}$ and NK cells that promoted angiostasis in the tumor by altering the balance of pro- and antiangiogenic chemokines. This suggests that MDSC depletion not only improves APC, NK cell, and T-cell immune activities but also promotes antiangiogenesis, leading to a more effective tumor control.

Although the depletion by antibodies affects other Gr1or Ly6G-expressing monocytes, the data suggest that the depletion of MDSCs is beneficial in eliciting anticancer effects and are consistent with studies by other groups..$^{47,48}$ Therapeutic vaccination led to decreased tumor burden without complete eradication of the tumors. Depletion of MDSCs in combination with the vaccine led to a 20 -fold reduction in tumor burden in $50 \%$ of the mice and to complete eradication of tumors in the remainder of animals. These findings suggest that an effective attack necessitates changes in multiple components of the immune system in parallel, leading to immune cell activation and to the disruption of the regulatory mechanisms that limit antitumor immune responses. MDSC depletion reprograms the tumor niche by altering the inflammatory responses, allowing tumor immune destruction and generation of immunological memory.

\section{Conclusion}

Despite the identification of a repertoire of tumor antigens, hurdles persist for immune-based anticancer therapies. Tumor-induced immune suppression contributes to the limited efficacy of the current approaches. More effective immunotherapeutic strategies will likely result from an improved understanding of the mechanisms that sustain tumor growth. It is likely that combined therapies that address the multiple immune deficits in cancer patients will be required for effective therapy. MDSCs play a major role in the suppression of T-cell activation and they sustain tumor growth, proliferation, and metastases. Regulation of MDSC recruitment, differentiation or expansion, and inhibition of the MDSC suppressive function with pharmacologic agents will be useful in the control of cancer growth and progression. Pharmacologic agents that regulate MDSCs may be more effective when combined with immunotherapies. Optimization of combined approaches that simultaneously downregulate MDSC suppressor pathways, restore APC immune-stimulating activity, and expand tumorreactive $\mathrm{T}$ cells will be useful in improving therapy.

\section{Acknowledgement}

This research was supported by the NIH National Center for Advancing Translational Science through UCLA CTSI Grant UL1TR000124.

\section{Disclosure}

The authors report no conflicts of interest in this work.

\section{References}

1. Jemal A, Siegel R, Xu J, Ward E. Cancer statistics, 2010. CA Cancer J Clin. 2010;60(5):277-300.

2. Young MR, Wright MA, Pandit R. Myeloid differentiation treatment to diminish the presence of immune-suppressive CD34+ cells within human head and neck squamous cell carcinomas. J Immunol. 1997; 159(2):990-996.

3. Kusmartsev SA, Kusmartseva IN, Afanasyev SG, Cherdyntseva NV. Immunosuppressive cells in bone marrow of patients with stomach cancer. Adv Exp Med Biol. 1998;451:189-194.

4. Kusmartsev SA, Ogreba VI. Suppressor activity of bone marrow and spleen cells in C57B1/6 mice during carcinogenesis induced by 7 , 12-dimethylbenz(a)anthracene. Eksp Onkol. 1989;11(5):23-26. Russian.

5. Condamine T, Gabrilovich DI. Molecular mechanisms regulating myeloid-derived suppressor cell differentiation and function. Trends Immunol. 2011;32(1):19-25.

6. Zea AH, Rodriguez PC, Atkins MB, et al. Arginase-producing myeloid suppressor cells in renal cell carcinoma patients: a mechanism of tumor evasion. Cancer Res. 2005;65(8):3044-3048.

7. Poschke I, Mougiakakos D, Hansson J, Masucci GV, Kiessling R. Immature immunosuppressive CD14+HLA-DR-/low cells in melanoma patients are Stat3hi and overexpress CD80, CD83, and DC-sign. Cancer Res. 2010;70(11):4335-4345.

8. Srivastava MK, Bosch JJ, Thompson JA, Ksander BR, Edelman MJ, Ostrand-Rosenberg S. Lung cancer patients' CD4(+) T cells are activated in vitro by MHC II cell-based vaccines despite the presence of myeloid-derived suppressor cells. Cancer Immunol Immunother. 2008;57(10):1493-1504.

9. Srivastava MK, Andersson $\AA$, Zhu L, et al. Myeloid suppressor cells and immune modulation in lung cancer. Immunotherapy. 2012;4(3): 291-304.

10. Gabrilovich DI, Nagaraj S. Myeloid-derived suppressor cells as regulators of the immune system. Nat Rev Immunol. 2009;9(3):162-174.

11. Chalmin F, Ladoire S, Mignot G, et al. Membrane-associated Hsp72 from tumor-derived exosomes mediates STAT3-dependent immunosuppressive function of mouse and human myeloid-derived suppressor cells. J Clin Invest. 2010;120(2):457-471.

12. Serafini P, Carbley R, Noonan KA, Tan G, Bronte V, Borrello I. Highdose granulocyte-macrophage colony-stimulating factor-producing vaccines impair the immune response through the recruitment of myeloid suppressor cells. Cancer Res. 2004;64(17):6337-6343. 
13. Song X, Krelin Y, Dvorkin T, et al. CD11b+/Gr-1+ immature myeloid cells mediate suppression of T cells in mice bearing tumors of IL-1 betasecreting cells. J Immunol. 2005;175(12):8200-8208.

14. Bunt SK, Sinha P, Clements VK, Leips J, Ostrand-Rosenberg S. Inflammation induces myeloid-derived suppressor cells that facilitate tumor progression. J Immunol. 2006;176(1):284-290.

15. Sinha P, Okoro C, Foell D, Freeze HH, Ostrand-Rosenberg S, Srikrishna G. Proinflammatory S100 proteins regulate the accumulation of myeloid-derived suppressor cells. J Immunol. 2008;181(7):4666-4675.

16. Cheng P, Corzo CA, Luetteke N, et al. Inhibition of dendritic cell differentiation and accumulation of myeloid-derived suppressor cells in cancer is regulated by S100A9 protein. J Exp Med. 2008;205(10): 2235-2249.

17. Corzo CA, Cotter MJ, Cheng P, et al. Mechanism regulating reactive oxygen species in tumor-induced myeloid-derived suppressor cells. J Immunol. 2009;182(9):5693-5701.

18. Mazzoni A, Bronte V, Visintin A, et al. Myeloid suppressor lines inhibit T cell responses by an NO-dependent mechanism. JImmunol.2002;168(2): 689-695.

19. Srivastava MK, Sinha P, Clements VK, Rodriguez P, OstrandRosenberg S. Myeloid-derived suppressor cells inhibit T-cell activation by depleting cystine and cysteine. Cancer Res. 2010;70(1):68-77.

20. Hanson EM, Clements VK, Sinha P, Ilkovitch D, Ostrand-Rosenberg S. Myeloid-derived suppressor cells down-regulate L-selectin expression on CD4+ and CD8+ T cells. J Immunol. 2009;183(2):937-944.

21. Nagaraj S, Gupta K, Pisarev V, et al. Altered recognition of antigen is a mechanism of CD8+ T cell tolerance in cancer. Nat Med. 2007;13(7): 828-835.

22. Nagaraj S, Collazo M, Corzo CA, et al. Regulatory myeloid suppressor cells in health and disease. Cancer Res. 2009;69(19):7503-7506.

23. Sakaguchi S. Regulatory T cells: key controllers of immunologic selftolerance. Cell. 2000;101(5):455-458.

24. Smyth MJ, Teng MW, Swann J, Kyparissoudis K, Godfrey DI, Hayakawa Y. CD4+CD25+T regulatory cells suppress NK cell-mediated immunotherapy of cancer. J Immunol. 2006;176(3):1582-1587.

25. Sinha P, Clements VK, Bunt SK, Albelda SM, Ostrand-Rosenberg S. Cross-talk between myeloid-derived suppressor cells and macrophages subverts tumor immunity toward a type 2 response. J Immunol. 2007; 179(2):977-983

26. Gabrilovich D, Pisarev V. Tumor escape from immune response: mechanisms and targets of activity. Curr Drug Targets. 2003;4(7): $525-536$.

27. Huang AY, Golumbek P, Ahmadzadeh M, Jaffee E, Pardoll D, Levitsky H. Role of bone marrow-derived cells in presenting MHC class I-restricted tumor antigens. Science. 1994;264(5161):961-965.

28. Albert ML, Sauter B, Bhardwaj N. Dendritic cells acquire antigen from apoptotic cells and induce class I-restricted CTLs. Nature. 1998; 392(6671):86-89.

29. Gerlini G, Tun-Kyi A, Dudli C, Burg G, Pimpinelli N, Nestle FO. Metastatic melanoma secreted IL-10 down-regulates CD1 molecules on dendritic cells in metastatic tumor lesions. Am J Pathol. 2004;165(6):1853-1863.

30. Gabrilovich DI, Chen HL, Girgis KR, et al. Production of vascular endothelial growth factor by human tumors inhibits the functional maturation of dendritic cells. Nat Med. 1996;2(10):1096-1103.

31. Liu C, Yu S, Kappes J, et al. Expansion of spleen myeloid suppressor cells represses NK cell cytotoxicity in tumor-bearing host. Blood. 2007; 109(10):4336-4342.

ImmunoTargets and Therapy

\section{Publish your work in this journal}

ImmunoTargets and Therapy is an international, peer-reviewed open access journal focusing on the immunological basis of diseases, potential targets for immune based therapy and treatment protocols employed to improve patient management. Basic immunology and physiology of the immune system in health, and disease will be also covered. In addition, the journal will focus on the impact of manage-

Submit your manuscript here: http://www.dovepress.com/immunotargets-and-therapy-journa
32. Huang M, Stolina M, Sharma S, et al. Non-small cell lung cancer cyclooxygenase-2-dependent regulation of cytokine balance in lymphocytes and macrophages: up-regulation of interleukin 10 and down-regulation of interleukin 12 production. Cancer Res. 1998;58(6): 1208-1216.

33. Stolina M, Sharma S, Lin Y, et al. Specific inhibition of cyclooxygenase 2 restores antitumor reactivity by altering the balance of IL-10 and IL-12 synthesis. J Immunol. 2000;164(1):361-370.

34. Sharma S, Stolina M, Yang SC, et al. Tumor cyclooxygenase 2-dependent suppression of dendritic cell function. Clin Cancer Res. 2003;9(3):961-968.

35. Dohadwala M, Batra RK, Luo J, et al. Autocrine/paracrine prostaglandin E2 production by non-small cell lung cancer cells regulates matrix metalloproteinase-2 and CD44 in cyclooxygenase-2-dependent invasion. J Biol Chem. 2002;277(52):50828-50833.

36. Rodriguez PC, Hernandez CP, Quiceno D, et al. Arginase I in myeloid suppressor cells is induced by COX-2 in lung carcinoma. J Exp Med. 2005;202(7):931-939.

37. Woo EY, Chu CS, Goletz TJ, et al. Regulatory CD4(+)CD25(+) T cells in tumors from patients with early-stage non-small cell lung cancer and late-stage ovarian cancer. Cancer Res. 2001;61(12):4766-4772.

38. Sharma S, Yang SC, Zhu L, et al. Tumor cyclooxygenase-2/ prostaglandin E2-dependent promotion of FOXP3 expression and $\mathrm{CD} 4+\mathrm{CD} 25+\mathrm{T}$ regulatory cell activities in lung cancer. Cancer Res. 2005;65(12):5211-5220.

39. Baratelli F, Lin Y, Zhu L, et al. Prostaglandin E2 induces FOXP3 gene expression and $\mathrm{T}$ regulatory cell function in human $\mathrm{CD} 4+\mathrm{T}$ cells. J Immunol. 2005;175(3):1483-1490.

40. Cano A, Pérez-Moreno MA, Rodrigo I, et al. The transcription factor Snail controls epithelial-mesenchymal transitions by repressing E-cadherin expression. Nat Cell Biol. 2000;2(2):76-83.

41. Jeanes A, Gottardi CJ, Yap AS. Cadherins and cancer: how does cadherin dysfunction promote tumor progression? Oncogene. 2008;27(55): 6920-6929.

42. Yanagawa J, Walser TC, Zhu LX, et al. Snail promotes CXCR2 liganddependent tumor progression in non-small cell lung carcinoma. Clin Cancer Res. 2009;15(22):6820-6829.

43. Kudo-Saito C, Shirako H, Takeuchi T, Kawakami Y. Cancer metastasis is accelerated through immunosuppression during Snail-induced EMT of cancer cells. Cancer Cell. 2009;15(3):195-206.

44. Srivastava MK, Sharma S, Zhu L, et al. Abstract A17: Tumor Snail knockdown reduces tumor burden and metastases by inducing antitumor immune responses in lung cancer. Cancer Res. 2011; 71(Suppl 18):A17.

45. Butts C, Murray N, Maksymiuk A, et al. Randomized phase IIB trial of BLP25 liposome vaccine in stage IIIB and IV non-small-cell lung cancer. J Clin Oncol. 2005;23(27):6674-6681.

46. Srivastava MK, Zhu L, Harris-White M, et al. Myeloid suppressor cell depletion augments antitumor activity in lung cancer. PLoS One. 2012; 7(7):e40677.

47. Mundy-Bosse BL, Lesinski GB, Jaime-Ramirez AC, et al. Myeloidderived suppressor cell inhibition of the IFN response in tumor-bearing mice. Cancer Res. 2011;71(15):5101-5110.

48. Fujita M, Kohanbash G, Fellows-Mayle W, et al. COX-2 blockade suppresses gliomagenesis by inhibiting myeloid-derived suppressor cells. Cancer Res. 2011;71(7):2664-2674.

ment programs and new therapeutic agents and protocols on patient perspectives such as quality of life, adherence and satisfaction. The manuscript management system is completely online and includes a very quick and fair peer-review system, which is all easy to use. Visit http://www.dovepress.com/testimonials.php to read real quotes from published authors. 